\begin{tabular}{|l|c|c|c|l|l|}
\hline \multirow{2}{*}{$\mathcal{L}$} & C O A & \multicolumn{1}{|c|}{$\mathbf{1 2 5 6 9}$} & \multirow{2}{*}{ WILEY } & Dispatch: 3.11 .15 & CE: Nivetha \\
\cline { 2 - 3 } \cline { 5 - 6 } & Journal Code & Manuscript No. & & No. of pages: & PE: Nagappan \\
\hline
\end{tabular}

\title{
Guidelines for quantification of noise-induced hearing loss in a medicolegal context
}

\author{
4 Lutman, M.E., ${ }^{*}$ Coles, R.R.A. ${ }^{\dagger}$ \& Buffin, J.T.* \\ ${ }^{*}$ Audiology, Institute of Sound and Vibration Research, University of Southampton, Southampton, ${ }^{\dagger}$ MRC Institute of Hearing Research, \\ University Park, Nottingham, ${ }^{\star}$ Department of Medical Audiology, Royal Hallamshire Hospital, Sheffield, UK \\ Accepted for publication 3 August 2015 \\ Clin. Otolaryngol. 2015, 00, 000-000
}

Objectives: Guidelines published in 2000 by the authors are widely used by medical and legal professionals in the UK for diagnosis of noise-induced hearing loss in a medicolegal context. However, they cannot be used for quantification of the noise-induced hearing loss, which is required in most cases. This requirement is addressed.

Design: A method is developed here to quantify noiseinduced hearing loss, thereby overcoming this shortcoming. Setting: Assessment of noise-induced hearing loss in medicolegal cases.
Participants: A consecutive series of 124 cases of noiseinduced hearing loss is used for evaluation.

Main outcome measure: Magnitude of noise-induced hearing loss based on hearing threshold levels averaged over the frequencies 1, 2 and $3 \mathrm{kHz}$.

Results: The rationale of the method, practical application and three worked examples are developed. A simpler shortcut method is developed and shown to be equivalent to the full method in most cases.

Conclusions: The method offers a practical approach to quantification of noise-induced hearing loss.

\section{Background}

In legal cases where claimants allege that their hearing has been damaged by noise exposure, usually at work, the claimant must prove both liability and causation. A major part of the causation argument is to show that their hearing loss has been caused by the alleged noise exposure, on the balance of probability. Guidance for medical experts has been provided by the Coles, Lutman and Buffin guidelines ${ }^{1}$ for diagnosis of noise-induced hearing loss, which are now widely used in the UK by both medical and legal professions in compensation claims for noise-induced hearing loss. The main underlying principle of the Guidelines is to identify a shape in the pure tone audiogram that is consistent with noise-induced hearing loss, essentially a notch or downward bulge in the frequency range $3-6 \mathrm{kHz}$. This derives from the plethora of scientific publications that show that noise damage tends to be evident as a notch centred on approximately $4 \mathrm{kHz}$ in many cases. However, with developing ageassociated hearing loss or other constitutional sensorineural hearing loss adding to noise-induced hearing loss, the notch

Correspondence: Mark Lutman, Institute of Sound and Vibration Research, University of Southampton, Highfield, Southampton SO17 1BJ, UK. Tel: 02380261505; Fax: XXXXXXX; e-mail: mel@isvr.soton.ac.uk tends to disappear, leaving the noise-induced hearing loss element as a bulge below the age-associated hearing loss.

In essence, the procedures defined in the Guidelines identify the notch or bulge by drawing a contour between anchor points at frequencies below and above the $3-6 \mathrm{kHz}$ region and setting criteria regarding the extent that the notch/bulge must descend below that contour in order to satisfy requirements for a probable diagnosis of noiseinduced hearing loss. However, the Guidelines state explicitly that the notch/bulge does not define the whole extent of the component of noise-induced hearing loss, because there will often be some noise-induced hearing loss at the anchor points, which are usually at the frequencies 1 and $8 \mathrm{kHz}$. Therefore, the Guidelines limit their scope to diagnosis and stop short of quantification of the noise-induced hearing loss component.

Approaches to quantification of noise-induced hearing loss vary. One approach ${ }^{2}$ simply assumes that age-associated hearing loss in the individual is represented by the median (50th percentile) age-associated hearing loss for men or women of the same age as the claimant. The authors of that approach accept that it involved 'rough justice' because the individual may have had age-associated hearing loss less than or greater than the 50th percentile. An alternative approach involves selecting a percentile that better matches the 2 individual's audiogram, based on fit to frequencies above 
and below the $3-6 \mathrm{kHz}$ range (i.e. frequencies that are less affected by noise); different percentiles might be adopted for the right and left ears. This approach is not satisfying if the lower and higher frequencies appear to correspond to widely different percentiles, which raises the question of which percentile to choose. A further step might be to tilt the ageassociated hearing loss percentile curves so that a better match to a single percentile was achieved at both lower and higher frequencies. This would be akin to derivation of the 'adjusted age-associated hearing loss values' in the above Guidelines.

The above sequence of approaches involves progressively greater customisation of standard age-associated hearing loss curves with the aim of estimating the underlying ageassociated hearing loss of the individual more accurately, thus allowing the residual noise-induced hearing loss component of the audiogram to be estimated in a more individualised fashion. This theme of customisation is explored further in the remainder of the article, whereby the main ${ }_{2}$ is to propose an extension to the Guidelines that allows the noise-induced hearing loss component to be estimated quantitatively.

\section{Quantification of noise-induced hearing loss}

As indicated above, quantification of the noise-induced hearing loss component of hearing loss is tantamount to quantification of the other components of hearing loss, mainly age-associated hearing loss. The actual hearing loss can be considered for practical purposes to be the sum of those components, including noise-induced hearing loss. Therefore, this article can be viewed as estimating ageassociated hearing loss in individual cases, recognising that the average pattern of age-associated hearing loss is an abstract notion that does not apply directly to many individuals. Not only is there a range of severity of ageassociated hearing loss, as recognised in standards such as ISO $7029^{3}$ by a range of percentiles, but also some people have a different shape to their age-associated hearing loss contour; for example, they may have more age-associated hearing loss than usual at lower frequencies and less at higher frequencies. Moreover, there may be additional hearing loss over and above age-associated hearing loss that is not attributable to noise-induced hearing loss and needs to be allowed for. For example, not infrequently there is a component of sensorineural hearing loss at low and midfrequencies that is greater than age-associated hearing loss (which is minimal at low and mid-frequencies); it cannot be attributable to noise-induced hearing loss as the frequencies are too low to be affected by noise when there is only mild or moderate noise-induced hearing loss. Such additional lowto mid-frequency hearing loss appears to occur commonly in older people. Using standardised age-associated hearing loss curves would fail to allow for such additional hearing loss; if standardised age-associated hearing loss values were simply subtracted from the measured hearing thresholds to estimate noise-induced hearing loss, the magnitude of noise-induced hearing loss would be inflated in the low- to mid-frequency region.

The original Guidelines make a rough estimate of ageassociated hearing loss by adopting anchor points usually at 1 and $8 \mathrm{kHz}$ and, as indicated above, recognise that the anchor points probably over-estimate age-associated hearing loss due to noise-induced hearing loss at the anchor points. However, if that over-estimation could be quantified, at least to an approximation, a more accurate estimate of ageassociated hearing loss could be obtained. In turn, by subtracting the more accurate estimate of age-associated hearing loss from the measured hearing loss, the noiseinduced hearing loss component itself could be quantified more accurately.

The procedure proposed here for quantification of the noise-induced hearing loss component comprises two passes: Pass One entails carrying out bulge calculations as per the original Guidelines methodology (even where the diagnosis of noise-induced hearing loss was based on a significant notch rather than a bulge). Pass Two is additional and involves the following four steps:

1 Estimate the extent to which the anchor points include some noise-induced hearing loss.

2 Make corresponding alterations to create modified anchor points.

3 Fit a modified age-associated hearing loss contour to the modified anchor points using the same approach as defined in the Guidelines.

4 Quantify noise-induced hearing loss as the difference between the measured thresholds and the modified ageassociated hearing loss contour (setting negative differences to zero).

These four steps are outlined in the following sections.

\section{Estimating the amount of noise-induced hearing loss at the anchor points}

Noise-induced hearing loss is generally greatest at $4 \mathrm{kHz}$ and less at higher and lower frequencies. This pattern is well established from meta-analysis of large studies of noiseinduced hearing loss in ISO $1999^{4,5}$, and in principle, it would be possible to determine the extent to which the anchor points over-estimate age-associated hearing loss by fitting appropriate patterns of noise-induced hearing loss from ISO 1999 to the measured hearing threshold levels. Unfortunately, ISO 1999 only estimates noise-induced hearing loss at frequencies up to $6 \mathrm{kHz}$; it does not provide 
estimates at $8 \mathrm{kHz}$, which is critical for the present purposes. The reason for the limitation of ISO 1999 is that the main studies of noise-induced hearing loss on which it is based (e.g. Burns and Robinson) ${ }^{6}$ only measured hearing thresholds up to $6 \mathrm{kHz}$. However, examination of other literature has allowed us to estimate noise-induced hearing loss at $8 \mathrm{kHz}$, as described in more detail in Appendix 1.

It turns out that the modifications required for the two anchor points can be accomplished by two simple formulae, based on the initially calculated noise-induced hearing loss bulge at $4 \mathrm{kHz}\left(N_{4 k}^{1}\right)$, which is obtained at Pass One by subtracting the adjusted age-associated hearing loss at $4 \mathrm{kHz}$ $\left(A_{4 k}^{1}\right)$ from the measured threshold at $4 \mathrm{kHz}$ (see worked examples below for clarification). For Pass Two, the modification to be applied to the $1 \mathrm{kHz}$ anchor point is given by $0.15 \times N_{4 k}^{1}$. Similarly, the modification to the $8 \mathrm{kHz}$ anchor point is given by $0.4 \times N_{4 k}^{1}$. An important restriction is that neither anchor point should be adjusted to become better than $0 \mathrm{~dB}$. Instances where either of the above formulae would yield an anchor point better than $0 \mathrm{~dB}$ are rare, but in such cases, the corresponding adjusted anchor point should be set to $0 \mathrm{~dB}$.

\section{Calculating the modified age-associated hearing loss contour}

Once the modified anchor points have been derived, the modified age-associated hearing loss contour is calculated using exactly the same procedure as in the Guidelines. First, an appropriate standard age-associated hearing loss data set is chosen from the relevant table in the Guidelines for men or women, to give an approximate fit to the modified anchor points. Note that for Pass Two an age-associated hearing loss statistic different from the one used at Pass One could be more appropriate. Modified misfit values are calculated at the anchor points and then calculated for intermediate frequencies by interpolation (see Appendix 2 regarding the method for interpolation). These new misfit values are used to modify the Pass Two age-associated hearing loss data set, which has the effect that the modified age-associated hearing loss curve intersects the modified anchor points.

\section{Quantification of noise-induced component}

Noise-induced hearing loss is calculated simply as the difference between the measured hearing thresholds and the modified age-associated hearing loss curve, with the caveat that any negative differences are set to zero. It would not make sense to have negative noise-induced hearing loss. Note that this method requires the upper anchor point to be set at $8 \mathrm{kHz}$. In principle, a similar approach could be derived when using $6 \mathrm{kHz}$ as the upper anchor point.
However, such an approach would be unstable because of the similarity in the amounts of noise-induced hearing loss at 4 and $6 \mathrm{kHz}$; there would be further instability introduced because of the additional variability observed in hearing threshold measurements at $6 \mathrm{kHz}$, especially when TDH-39 earphones are used.

\section{Worked examples}

Figure 1 shows an example audiogram (right ear only for simplicity) for a 65-year-old man demonstrating the typical $4-\mathrm{kHz}$ notch feature of noise-induced hearing loss. In Pass One, the audiogram is compared with the estimated ageassociated hearing loss values obtained using the original diagnostic Guidelines; in Pass Two, it is compared with the modified age-associated hearing loss values as proposed here. Table 1 shows the calculations that were required to obtain these two age-associated hearing loss sets of values. Table 1 also quantifies the noise-induced hearing loss component, as described above. The first block of the table (Pass One) follows the method of the Guidelines. The second block (Pass Two) first adjusts the lower anchor point by $3 \mathrm{~dB}$ from 15 to $12 \mathrm{~dB}$ and the upper anchor point by $9 \mathrm{~dB}$, from 55 to $46 \mathrm{~dB}$. The value of $3 \mathrm{~dB}$ is obtained by multiplying the $23 \mathrm{~dB}$ estimated noise-induced hearing loss at $4 \mathrm{kHz}$ in the

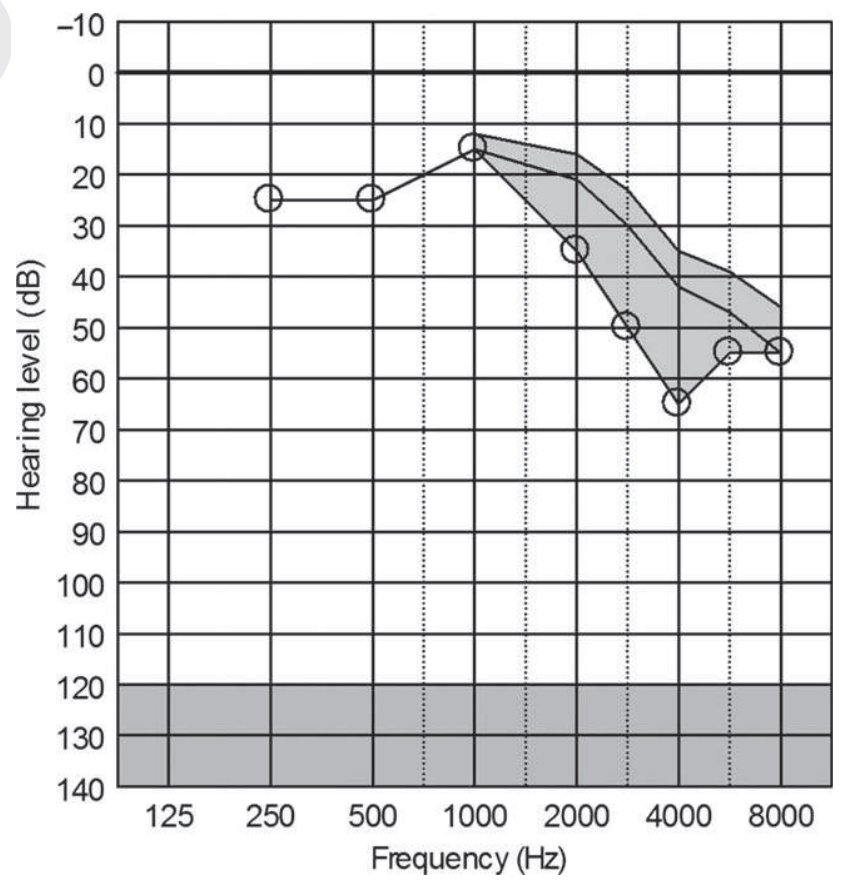

Fig. 1. Example audiogram for right ear only (circles), ageassociated hearing loss calculated according to guidelines (lower line) and modified age-associated hearing loss according to this study (upper line). The shaded area between the audiogram and the upper line represents the estimated noise-induced hearing loss. 
Table 1. Worked Example A

\begin{tabular}{|c|c|c|c|c|c|c|c|c|}
\hline & \multicolumn{8}{|c|}{ Frequency $(\mathrm{kHz})$} \\
\hline & 0.25 & 0.5 & 1 & 2 & 3 & 4 & 6 & 8 \\
\hline \multicolumn{9}{|l|}{ Pass One } \\
\hline Hearing threshold level (dB) & 25 & 25 & 15 & 35 & 50 & 65 & 55 & 55 \\
\hline HTL at selected anchor points $(\mathrm{dB})$ & & & $15^{\S}$ & & & & & $55^{\star *}$ \\
\hline Selected age-associated hearing loss statistic ${ }^{\star}(\mathrm{dB})$ & & & 11 & 19 & 28 & 41 & 47 & 56 \\
\hline Misfit values $(\mathrm{dB})$ & & & 4 & & & & & -1 \\
\hline Interpolated misfit values $(\mathrm{dB})$ & & & 4 & 2 & 2 & 1 & 0 & -1 \\
\hline Adjusted age-associated hearing loss values ( $\mathrm{dB}$ ) & & & 15 & 21 & 30 & $42^{\dagger}$ & 47 & 55 \\
\hline Bulge $(\mathrm{dB})$ & & & $\mathbf{0}$ & 14 & 20 & $23^{*}$ & 8 & $\mathbf{0}$ \\
\hline \multicolumn{9}{|l|}{ Pass Two } \\
\hline Modified HTL at anchor points (see text) & & & $12^{q}$ & & & & & $46^{\dagger \dagger}$ \\
\hline Selected age-associated hearing loss statistic ${ }^{\star}(\mathrm{dB})$ & & & 11 & 19 & 28 & 41 & 47 & 56 \\
\hline Misfit values $(\mathrm{dB})$ & & & 1 & & & & & -10 \\
\hline Interpolated misfit values $(\mathrm{dB})$ & & & 1 & -3 & -5 & -6 & -8 & -10 \\
\hline Modified age-associated hearing loss values (dB) & & & 12 & 16 & 23 & 35 & 39 & 46 \\
\hline $\begin{array}{l}\text { Modified bulge }(\mathrm{dB})=\text { noise-induced } \\
\text { hearing loss component }\end{array}$ & & & 3 & 19 & 27 & 30 & 16 & 9 \\
\hline
\end{tabular}

*50th percentile aged 65 years.

${ }^{\dagger} A_{4 k}^{1}$ in text.

${ }^{\ddagger} N_{4 k}^{1}$ in text.

${ }^{\S}$ Unmodified anchor point at $1 \mathrm{kHz}$.

$\mathbf{S}^{=} \epsilon_{\mathbf{L}}-\left(0.15 \times N_{4 k}^{1}\right)$.

${ }^{*}$ Unmodified anchor point at $8 \mathrm{kHz}$.

${ }^{\dagger \dagger}=\mathrm{e}_{-}-\left(0.4 \times N_{4 k}^{1}\right)$.

first block of the table by 0.15 and rounding to the nearest decibel; $9 \mathrm{~dB}$ is obtained by multiplying the estimated noiseinduced hearing loss at $4 \mathrm{kHz}$ by 0.4 and rounding to the nearest decibel. The remainder of the second block of the table follows the same process as the first block, although the numerical values are of course different because the anchors points have been modified. Note that in some cases, the selected age-associated hearing loss statistic may be different in Pass Two (see Worked Example C; Table 3 and Fig. 3 ).

Figures 2 and 3 show further worked examples in the same format as Figure 1, with the corresponding calculations in Tables 2 and 3. It can be seen that the amount of adjustment of the anchor points depends on the extent of noise-induced hearing loss; for small components of noiseinduced hearing loss, the adjustments are small (Fig. 2), while for larger components, the adjustment is larger (Fig. 3). When there is a deep notch at $4 \mathrm{kHz}$, there is the possibility that there may be an overcorrection of ageassociated hearing loss. In the example shown in Fig. 3, ageassociated hearing loss is probably overcorrected and the noise-induced hearing loss component is probably overestimated as a consequence. Note that the adjustments of the anchor points are independent of age (and hence ageassociated hearing loss).
The authors propose this method of quantification for use by medical expert witnesses and by the Courts generally as providing more scientifically based and realistic estimates of the amount of age-associated hearing loss and noise-induced hearing loss than hitherto available.

Inevitably, some cases will be somewhat underestimated by the method and others somewhat overestimated, and thus, there remains a need for the medical expert to cast a critical eye over the estimate before reaching a final conclusion. Nonetheless, it is considered that the method of quantification proposed here will reduce unnecessary variation among experts and provide a scientifically based procedure for quantification of noise damage.

\section{Short-cut method}

It is recognised that the two-pass approach described above involves extra work, compared to the original Guidelines. In Appendix 1, a short-cut method is suggested that is probably sufficient for most cases and simply involves a minor extension to the original Guidelines method. It is suitable when the aim is restricted to quantifying noise-induced hearing loss by means of the conventional $1-2-3-\mathrm{kHz}$ average. As described in Appendix 1, the short-cut method 


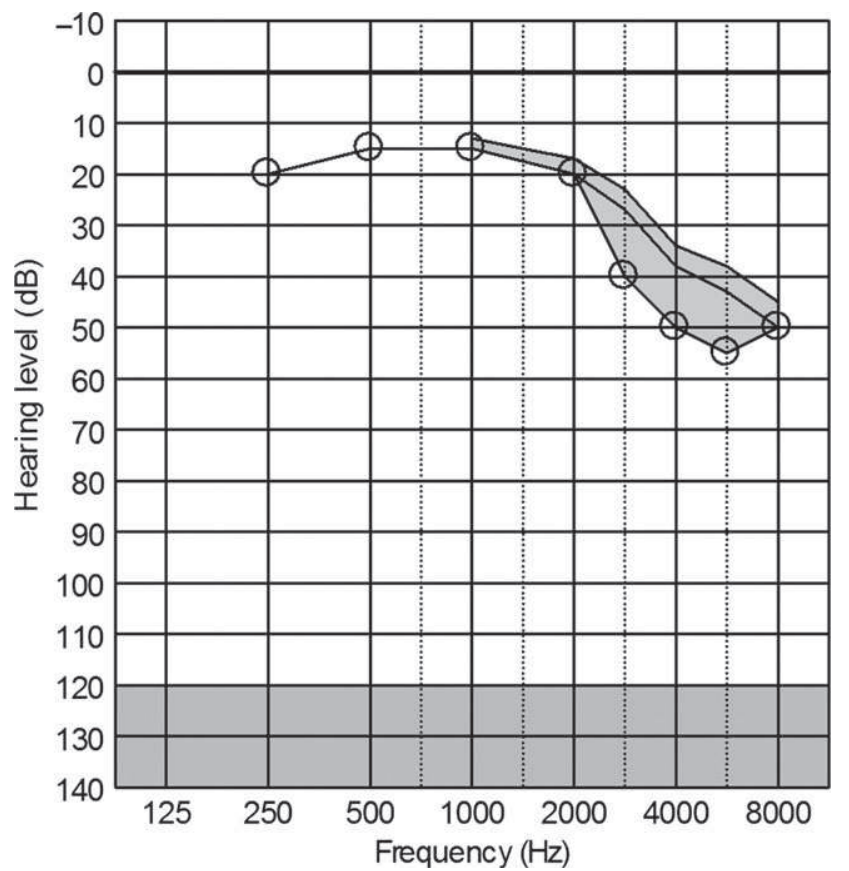

Fig. 2. Further example audiogram in same format as Fig. 1. Note there is less correction of age-associated hearing loss than if Fig. 1 because of there is a shallower bulge at $4 \mathrm{kHz}$ (see Table 2 for numerical data).

entails taking the average of the Pass One bulge row values at 1,2 and $3 \mathrm{kHz}$ (after setting any negative values to zero); this average is then increased by a factor of one-third to account for over-estimation of age-associated hearing loss at the anchor points. A binaural estimate can be derived by performing the above calculation for each ear and taking four-fifths of the smaller average plus one-fifth of the larger average. As indicated in Appendix 1, when there is a deep notch at $4 \mathrm{kHz}$ or when the maximum noise-induced hearing loss is at a frequency below $4 \mathrm{kHz}$, it may be preferable to perform the full method.

\section{Discussion}

There is no way to determine exactly the extent of noiseinduced hearing loss in individuals from simple measurement of hearing loss after the event. Even with careful longitudinal measurement of hearing throughout the period of noise exposure, determination of the extent of noiseinduced hearing loss is only approximate, because developing age-associated hearing loss occurring in the individual is confounded with developing noise-induced hearing loss. Therefore, quantification of noise-induced hearing loss is at best an indirect estimate. The estimation procedures recommended here should be considered in that context to be an approximation. Further, although the procedure is

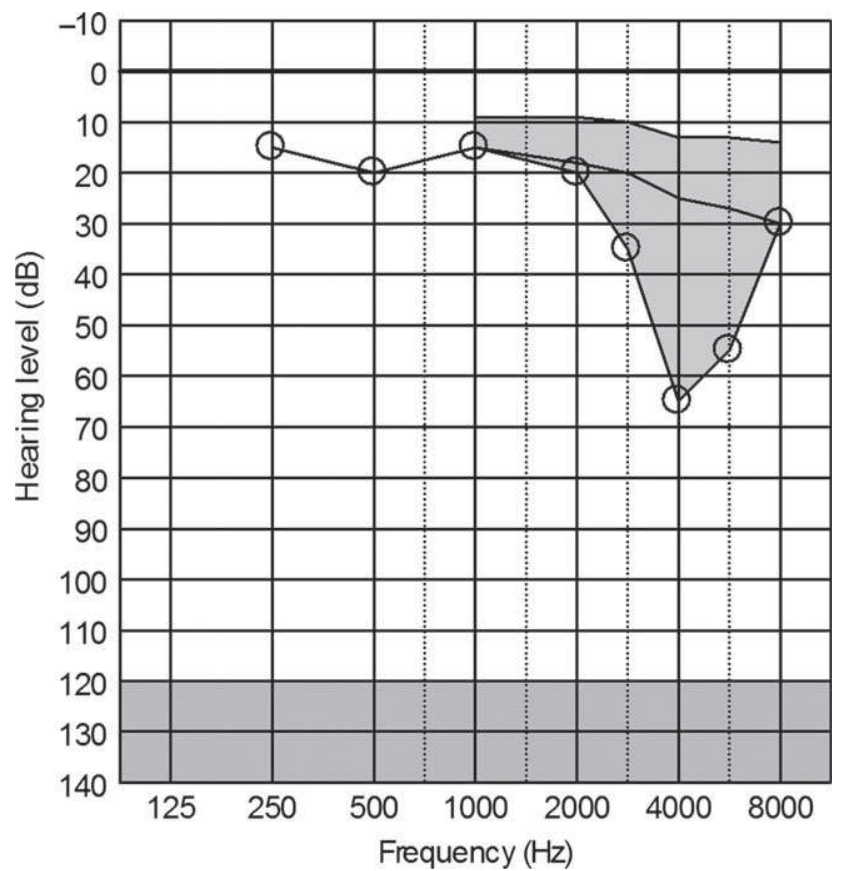

Fig. 3. Further example audiogram in same format as Fig. 1. Note there is greater correction of age-associated hearing loss than if Fig. 1 because of the steeper notch at $4 \mathrm{kHz}$. In this instance, there may be over-correction of age-associated hearing loss (see Appendix 1) (see Table 3 for numerical data).

founded on substantial and well-established literature on noise-induced hearing loss, the literature is necessarily limited by the range of noise exposures within the underlying studies and variation among the individuals participating. It is also based on median data from large groups to characterise noise-induced hearing loss and exposure duration of 10 years. If a longer duration ( 40 years) had been used as the basis on the noise-induced hearing loss model, the simple scaling approximation (Appendix 1) would have been inaccurate for the highest noise level of $100 \mathrm{~dB}(\mathrm{~A})$. Moreover, the underlying research dates back several decades because opportunities to gather data from humans exposed to high noise levels without hearing protection for long periods have virtually disappeared in developed countries. Therefore, the state of knowledge on noise-induced hearing loss in humans is unlikely to advance greatly in the near future. These limitations should be borne in mind when using the proposed method.

In cases where noise-induced hearing loss is mainly at frequencies other than $4 \mathrm{kHz}$, such as $3 \mathrm{kHz}$ or $6 \mathrm{kHz}$, the modification of anchor points at Pass Two will probably be too low, because the modification is based on the approximate noise-induced hearing loss at $4 \mathrm{kHz}$ obtained at Pass One. The consequence is that age-associated hearing loss will be overestimated somewhat, and hence, noise-induced 
Table 2. Worked Example B

\begin{tabular}{|c|c|c|c|c|c|c|c|c|}
\hline & \multicolumn{8}{|c|}{ Frequency $(\mathrm{kHz})$} \\
\hline & 0.25 & 0.5 & 1 & 2 & 3 & 4 & 6 & 8 \\
\hline \multicolumn{9}{|l|}{ Pass One } \\
\hline Hearing threshold level $(\mathrm{dB})$ & 20 & 15 & 15 & 20 & 40 & 50 & 55 & 50 \\
\hline HTL at selected anchor points $(\mathrm{dB})$ & & & 15 & & & & & 50 \\
\hline Selected age-associated hearing loss statistic ${ }^{*}(\mathrm{~dB})$ & & & 11 & 19 & 28 & 41 & 47 & 56 \\
\hline Misfit values $(\mathrm{dB})$ & & & 4 & & & & & -6 \\
\hline Interpolated misfit values $(\mathrm{dB})$ & & & 4 & 1 & -1 & -3 & -4 & -6 \\
\hline Adjusted age-associated hearing loss values (dB) & & & 15 & 20 & 27 & 38 & 43 & 50 \\
\hline Bulge $(\mathrm{dB})$ & & & $\mathbf{0}$ & $\mathbf{0}$ & 13 & 12 & 12 & $\mathbf{0}$ \\
\hline \multicolumn{9}{|l|}{ Pass Two } \\
\hline Modified HTL at anchor points (see text) & & & 13 & & & & & 45 \\
\hline Selected age-associated hearing loss statistic ${ }^{\star}(\mathrm{dB})$ & & & 11 & 19 & 28 & 41 & 47 & 56 \\
\hline Misfit values $(\mathrm{dB})$ & & & 2 & & & & & -11 \\
\hline Interpolated misfit values $(\mathrm{dB})$ & & & 2 & -2 & -5 & -7 & -9 & -11 \\
\hline Modified age-associated hearing loss values (dB) & & & 13 & 17 & 23 & 34 & 38 & 45 \\
\hline $\begin{array}{l}\text { Modified bulge }(\mathrm{dB})=\text { noise-induced } \\
\text { hearing loss component }\end{array}$ & & & 2 & 3 & 17 & 16 & 17 & 5 \\
\hline
\end{tabular}

*50th percentile aged 65 years.

Table 3. Worked Example C

\begin{tabular}{|c|c|c|c|c|c|c|c|c|}
\hline & \multicolumn{8}{|c|}{ Frequency $(\mathrm{kHz})$} \\
\hline & 0.25 & 0.5 & 1 & 2 & 3 & 4 & 6 & 8 \\
\hline \multicolumn{9}{|l|}{ Pass One } \\
\hline Hearing threshold level (dB) & 15 & 20 & 15 & 20 & 35 & 65 & 55 & 30 \\
\hline HTL at selected anchor points (dB) & & & 15 & & & & & 30 \\
\hline Selected age-associated hearing loss statistic ${ }^{*}(\mathrm{~dB})$ & & & 9 & 14 & 17 & 24 & 27 & 31 \\
\hline Misfit values $(\mathrm{dB})$ & & & 6 & & & & & -1 \\
\hline Interpolated misfit values $(\mathrm{dB})$ & & & 6 & 4 & 3 & 1 & 0 & -1 \\
\hline Adjusted age-associated hearing loss values (dB) & & & 15 & 18 & 20 & 25 & 27 & 30 \\
\hline Bulge $(\mathrm{dB})$ & & & $\mathbf{0}$ & 2 & 15 & 40 & 28 & $\mathbf{0}$ \\
\hline \multicolumn{9}{|l|}{ Pass Two } \\
\hline Modified HTL at anchor points (see text) & & & 9 & & & & & 14 \\
\hline Selected age-associated hearing loss statistic ${ }^{\star *}(\mathrm{~dB})$ & & & 4 & 7 & 9 & 14 & 16 & 18 \\
\hline Misfit values $(\mathrm{dB})$ & & & 5 & & & & & -4 \\
\hline Interpolated misfit values $(\mathrm{dB})$ & & & 5 & 2 & 1 & -1 & -3 & -4 \\
\hline Modified age-associated hearing loss values (dB) & & & 9 & 9 & 10 & 13 & 13 & 14 \\
\hline $\begin{array}{l}\text { Modified bulge }(\mathrm{dB})=\text { noise-induced hearing } \\
\text { loss component }\end{array}$ & & & 6 & 11 & 25 & 52 & 42 & 16 \\
\hline
\end{tabular}

${ }^{\star} 25$ th percentile aged 40 years.

$* * 50$ th percentile aged 40 years.

hearing loss will be underestimated. While this situation is not idea $a_{2}$ it is no worse than (and generally somewhat better than) simply using the bulge row without modification of the anchor points. As such, the proposed method is still an improvement on the common practice of using a fixed percentile for all frequencies and certainly better than simply using the 50th percentile. Moreover, where exposure has involved very high noise levels (daily personal noise exposure levels greater than $105 \mathrm{~dB}(\mathrm{~A}))$, the shape of the audiogram may differ from the model that underlies the present method. It follows that the method should not be applied directly in such cases.

With increasing age and therefore increasing age-associated hearing loss, there is a tendency for noise-induced hearing loss to be subsumed by age-associated hearing loss. This is reflected in the main formula of ISO 1999, where 
overall hearing loss is equated to the sum of age-associated hearing loss and noise-induced hearing loss minus a term based on the product of age-associated hearing loss and noise-induced hearing loss. The latter term is small when the overall hearing loss is less than $40 \mathrm{~dB}$ and increases for greater hearing loss. This means that the magnitude of noiseinduced hearing loss appears to reduce as people get older. The present approach to quantification reflects the current additional hearing loss due to noise and does not attempt to infer the amount of noise-induced hearing loss that may have been present in the past. That is appropriate where the aim is to provide guidance on the impact that noise-induced hearing loss would have at the time of examination.

\section{Concluding remarks}

In a medicolegal context, the expert witness is required to assist the Court by making best estimates when there is no alternative. One approach to quantification of loss of amenity is to simply compare the individual with the 'average person' (of the same age and sex). However, that approach is bound to incur a substantial element of 'rough justice', as indicated above, because individuals seldom conform to the average. In cases of noise-induced hearing loss, a person who had unusually good hearing, but for noise injury, would be disadvantaged and correspondingly a person with intrinsically poor hearing would gain an advantage. The procedure described here is aimed to reduce such advantages and disadvantages. Moreover, approaches based on comparing the individual with a particular ageassociated hearing loss percentile are prone to providing an inappropriate baseline at either lower frequencies or higher frequencies, or both when the age-associated hearing loss percentile only matches in the middle. The procedure described here will reduce those shortcomings as well. However, while considered to be an improvement on previous approaches, there remain some unavoidable limitations outlined above and it is recognised that the ultimate estimate of noise-induced hearing loss is no better than that:

4 an estimate,

\section{Conflict of interest}

None for second and third authors; the first author is still involved as an expert witness in medicolegal work, instructed equally by claimants and defendants.

\section{References}

1 Coles R.R.A., Lutman M.E. \& Buffin J.T. (2000) Guidelines on the diagnosis of noise-induced hearing loss for medicolegal purposes. Clin. Otolaryngol. 25, 264-273
2 King P.F., Coles R.R.A., Lutman M.E. et al. (1992) Assessment of Hearing Disability: Guidelines for Medicolegal Practice. Whurr, London

3 ISO 7029 (2000) Acoustics - Statistical Distribution of Hearing Thresholds as a Function of Age. International Organization for Standardization, Geneva

4 ISO 1999 (1990) Acoustics: Determination of Occupational Noise Exposure and Estimation of Noise-Induced Hearing Impairment. International Organization for Standardization, Geneva

5 ISO 1999 (2013) Acoustics: Estimation of Noise-Induced Hearing Loss. International Organization for Standardization, Geneva

6 Burns W. \& Robinson D.W. (1970) Noise in Industry. HMSO, London

7 Robinson D.W. \& Shipton M.S. (1977) Tables for the Estimation of Noise-Induced Hearing Loss. Report Ac 61. National Physical Laboratory, Teddington.

8 Passchier-Vermeer W. (1974) Hearing loss due to continuous exposure to steady-state broad-band noise. J. Acous. Soc. Am. 56, $1585-1593$

\section{Appendix 1}

\section{Modelling noise-induced hearing loss}

The main aim of the exercise described here is to estimate the contribution to noise-induced hearing loss at 1 and $8 \mathrm{kHz}$, based on a working estimate of noise-induced hearing loss at $4 \mathrm{kHz}$, which is the frequency that generally demonstrates the maximum noise-induced hearing loss. These values can then be used to obtain a better estimate of age-associated hearing loss and hence, by subtraction, a better indication of noise-induced hearing loss.

A necessary requirement for the exercise is a model of noise-induced hearing loss. One obvious contender is the international standard ISO $1999^{4,5}$, which is based on metaanalysis of three large studies. However, ISO 1999 is unsuitable because it does not include the frequency $8 \mathrm{kHz}$; that frequency was not included in the studies forming the basis for the meta-analysis. For the same reasons, the NPL Tables of Robinson and Shipton ${ }^{7}$ are not suitable. Fortunately, the meta-analysis of Passchier-Vermeer ${ }^{8}$ includes $8 \mathrm{kHz}$ and it is used here as the basis of a model of noise-induced hearing loss.

Unlike ISO 1999 and the NPL Tables, the PasschierVermeer study does not propose a mathematical model of noise-induced hearing loss and the following is based on analysis of data from Passchier-Vermeer's publication. Specifically, Fig. 6 from the publication is used, which plots $\mathbf{5}$ fitted curves relating median noise-induced hearing loss after 10 years of exposure to noise level. There are seven curves for the frequencies $0.5,1,2,3,4,6$ and $8 \mathrm{kHz}$. The curves have been used to extract four median noise-induced hearing loss patterns, specifically median (age-corrected) noise-induced hearing loss after 10 years of exposure to noise levels of 85 , 92, 97 and $102 \mathrm{~dB}(\mathrm{~A})$, as shown in Fig. 4. These points were 


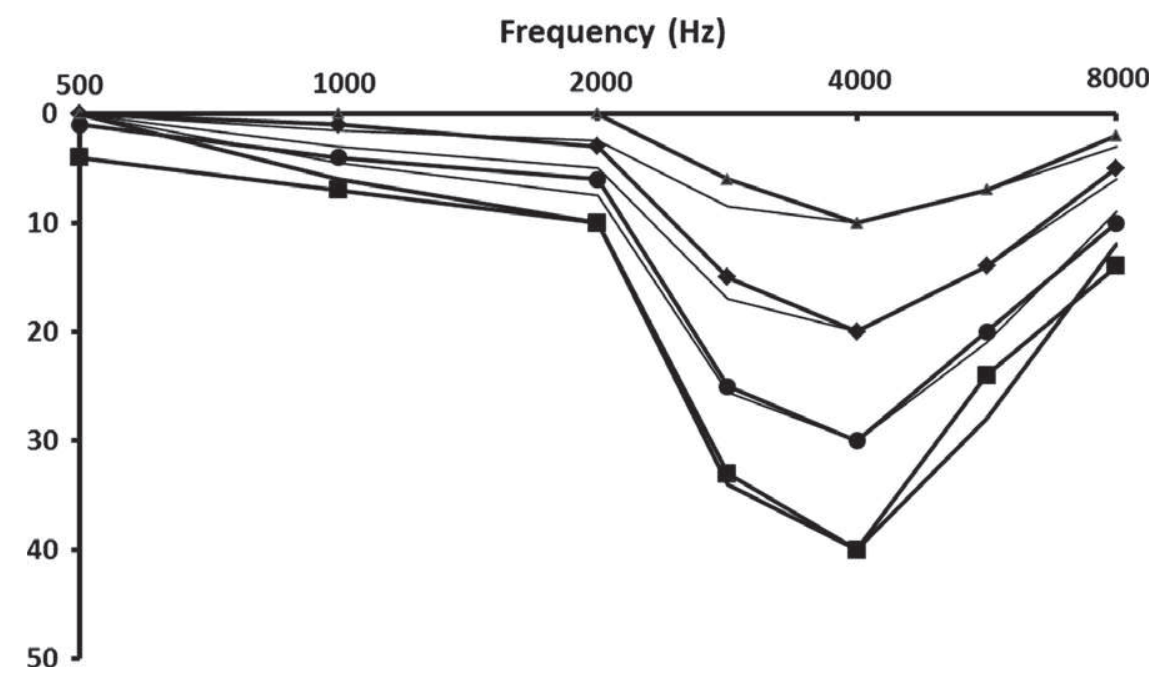

Fig. 4. Noise-induced hearing loss curves based on Passchier-Vermeer ${ }^{8}$. The vertical axis is age-corrected hearing threshold level in $\mathrm{dB}$. Thick lines with symbols (from top to bottom) are for daily personal noise exposure levels for 10 years of 85, 92, 97 and $100 \mathrm{~dB}(\mathrm{~A})$ ). Thin lines are for simple scaling model (see text).

chosen from the curves because they correspond to noiseinduced hearing loss at $4 \mathrm{kHz}$ of 10, 20, 30 and $40 \mathrm{~dB}$. The plots show that noise-induced hearing loss is maximal at $4 \mathrm{kHz}$, as expected, and inspection suggests that their shapes are similar; to an approximation they could be related to one another by a simple scaling factor; an exception to this is the curves for noise levels of 85 and $92 \mathrm{~dB}(\mathrm{~A})$, where there is less noise-induced hearing loss at 1 and $2 \mathrm{kHz}$ than predicted by the simple scaling model. The extent to which the plots are modelled by simply scaling a single curve can be seen by comparing the plots with the corresponding thin lines in Fig. 4; the thin lines represent a simple scaling model. Overall, the simple scaling model provides a good fit to the median data from the Passchier-Vermeer study. For the present purposes, an exact fit is unimportant.

The simple scaling model shown by the thin lines in Fig. 4 is defined as follows. Noise-induced hearing loss at 1 , 2, 3, 6 and $8 \mathrm{kHz}$ is obtained by multiplying noise-induced hearing loss at $4 \mathrm{kHz}$ by $0.10,0.25,0.85,0.7$ and 0.3 , respectively. This model is used as the basis for the following method.

When calculating the misfit values using the original Guidelines method, age-associated hearing loss at the anchor points is over-estimated according to the amount of noiseinduced hearing loss at 1 and $8 \mathrm{kHz}$. If the amount of noiseinduced hearing loss at $4 \mathrm{kHz}$ is $N_{4 k}$ (not yet known), the over-estimation of age-associated hearing loss at $1 \mathrm{kHz}$ is $N_{4 k} \times 0.1$ and the over-estimation at $8 \mathrm{kHz}$ is $N_{4 k} \times 0.3$. It follows by interpolation (see Appendix 2 for method of interpolation) that the over-estimation at the intermediate frequencies 2, 3, 4 and $6 \mathrm{kHz}$ is $N_{4 k} \times 0.167, N_{4 k} \times 0.200$,
$N_{4 k} \times 0.233$ and $N_{4 k} \times 0.267$. Note that the over-estimation by $N_{4 k} \times 0.233$ at $4 \mathrm{kHz}$ means that the apparent noiseinduced hearing loss at $4 \mathrm{kHz}$ (bulge line) using the original Guidelines method is $N_{4 k} \times(1-0.233)$, or $N_{4 k} \times 0.767$. It follows that $N_{4 k}$ could be obtained from bulge line of the original method by multiplying the bulge value at $4 \mathrm{kHz}$ by $1 / 0.767=1.30$. By the same logic, the over-estimation of age-associated hearing loss at $1 \mathrm{kHz}$ can be obtained from the bulge value at $4 \mathrm{kHz}$ by multiplying by $0.1 / 0.767=0.13$. Similarly, the over-estimation of age-associated hearing loss at $8 \mathrm{kHz}$ can be obtained from the bulge value at $4 \mathrm{kHz}$ by multiplying by $0.3 / 0.767=0.39$.

The two factors required for the quantification of noiseinduced hearing loss are therefore 0.13 and 0.39 , to be applied at 1 and $8 \mathrm{kHz}$. However, as they are only approximate, we recommend using rounded values of 0.15 and 0.4 (see main text for their application).

The above factors are dependent on the approximate fit of the model to the Passchier-Vermeer data and also on the representativeness of the Passchier-Vermeer meta-analysis. However, in the present context, plausible variation of the factor values only makes small differences to the estimated noise-induced hearing loss.

It should be apparent that the method depends on using $8 \mathrm{kHz}$ as the upper anchor point frequency. In some cases, the threshold at $8 \mathrm{kHz}$ is clearly unrepresentative of ageassociated hearing loss and the original Guidelines allowed the use of $6 \mathrm{kHz}$ instead. However, experience of using $6 \mathrm{kHz}$ as an upper anchor point has shown that it can lead to anomalous outcomes and an alternative approach is recommended (see Appendix 2). 


\section{Short-cut method}

It is conventional in the UK to quantify noise-induced hearing loss using the average of values at the frequencies 1,2 and $3 \mathrm{kHz}$. It is also conventional to calculate the binaural average by adding four-fifths of the better-ear average to onefifth of the worse-ear average. Given the scaling model used above, it should be apparent that the adjustment to the anchor points is a fixed ratio of $N_{4 k}$ and therefore, a similar effect could be achieved by simply multiplying the bulge row values from Pass One of the calculations by a fixed ratio. That would eliminate the need to carry out the Pass Two calculations. It can be shown that, if the above scaling model is assumed to apply, the $1-2-3-\mathrm{kHz}$ average noise-induced hearing loss estimated by the full method can be derived from the bulge row $1-2-3-\mathrm{kHz}$ average from Pass One by simply multiplying by 1.32 . It follows that a simple expedient is to calculate the $1-2-3-\mathrm{kHz}$ average from the bulge row in Pass One (after setting any negative values to zero) and then add one-third. This is referred to here as the short-cut method.

To evaluate how the short-cut method works in practice, a consecutive series of 124 cases was examined and calculations performed using the full and the short-cut methods. The cases were all claimants submitted for assessment to the first author and had satisfied the original Guidelines audiometric requirements for a diagnosis of noise-induced hearing loss, on the balance of probability. Analyses were performed separately for right ear, left ear, better ear, worse ear and for the binaural average defined as above. Bivariate linear regression analysis was performed with the fullmethod estimate as the dependent variable and the 1-2-3-
$\mathrm{kHz}$ average bulge from Pass One as the independent variable. The analysis showed a high correlation in each analysis, with correlation coefficients ranging from 0.92 to 0.95 . Regression coefficients ranged from 1.33 to 1.37 , which is close to the theoretical value of 1.32 . The scatter plot in Fig. 5 shows the predictive relationship for the binaural 1-2$3-\mathrm{kHz}$ average. Further analysis of the data in Fig. 5 identified 17 of $124(14 \%)$ cases where there was a difference between the short-cut method and the full method of $2 \mathrm{~dB}$ or more. In 13 of the 17 cases, there was a deep notch at $4 \mathrm{kHz}$ and the full method gave a larger estimate than the short-cut method, and hence, the modification of the anchor points was relatively large. Arguably, the modification may have over-corrected age-associated hearing loss in some of the cases and therefore, the full method may have over-estimated noise-induced hearing loss (see Fig. 3 in main body of paper). When there is a deep notch at $4 \mathrm{kHz}$, it is desirable to use the full method and to plot the estimated age-associated hearing loss on the audiogram, so that it can be examined critically for possible over-correction. In the other 4 cases, hearing loss at $4 \mathrm{kHz}$ was less than at $3 \mathrm{kHz}$ and the anchor points may have been under-corrected. Where the maximum bulge value in Pass One is below $4 \mathrm{kHz}$, it may also be desirable to use the full method and review the ageassociated hearing loss estimate critically.

\section{Appendix 2}

\section{Required modifications of diagnostic guidelines}

The following modifications to the Guidelines are necessary to allow the proposed quantification of noise-induced

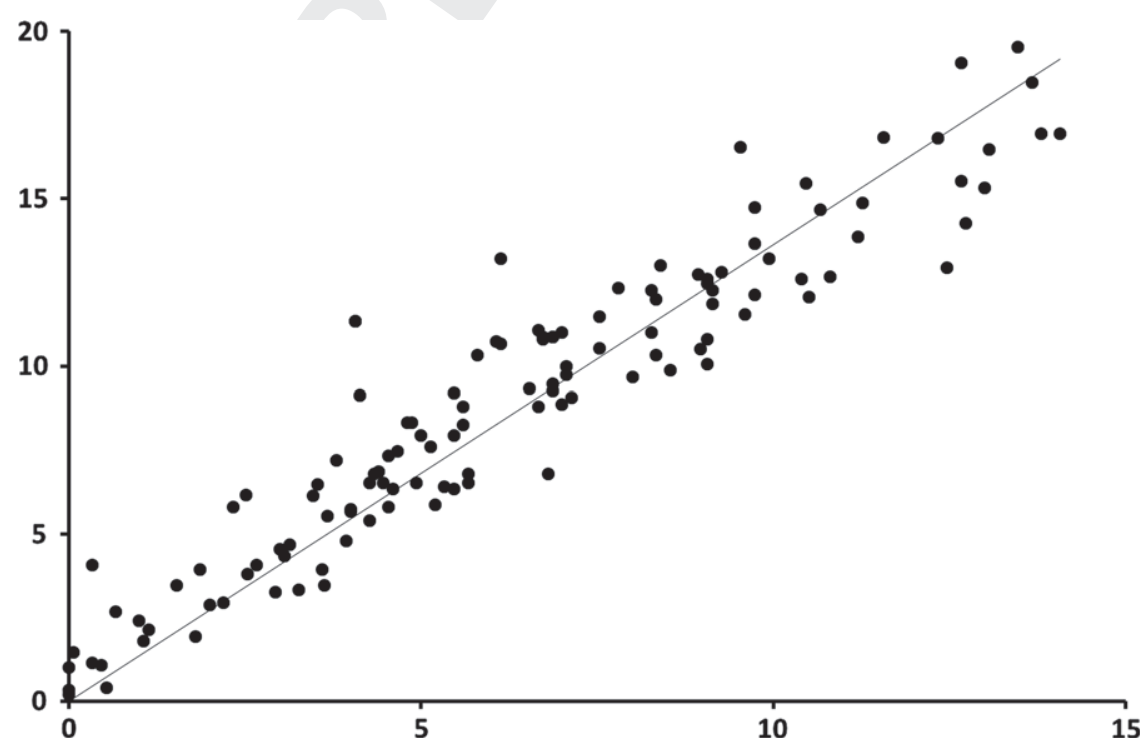

Fig. 5. Scatterplot showing relationship between estimated noise-induced hearing loss (binaural $1-2-3-\mathrm{kHz}$ average) for the full method and the short-cut method (see text for explanation of methods). Each symbol represents one case $(n=124)$. The line is the best-fit linear regression (slope 1.36, correlation coefficient 0.93 ). 
hearing loss to be used in a uniform fashion. To an extent, the modifications here involve clarification of the Guidelines on points that were hitherto ambiguous.

\section{Interpolation of misfit values}

The original Guidelines require the user to determine 'misfit' values at the anchor points (usually 1 and $8 \mathrm{kHz}$ ). The misfit values are the differences between the measured thresholds and the selected percentile for age-associated hearing loss at the anchor-point frequencies. Then, in order to estimate the misfit values at intermediate frequencies, the user is required to use interpolation. No formula for interpolation is given in the Guidelines and unfortunately the examples in the Appendix contained errors, making it impossible for the reader to infer what was intended. In order that the calculations in Appendix 1 apply, it is necessary to specify the method of interpolation.

Experience has shown that users of the Guidelines tend to use one of two possible methods. The first method considers the thresholds at 1, 2, 3, 4, 6 and $8 \mathrm{kHz}$ as a series of equally spaced numbers. As an example, if the misfit values at 1 and $8 \mathrm{kHz}$ were 0 and $15 \mathrm{~dB}$ respectively, the interpolated values at 2, 3, 4 and $6 \mathrm{kHz}$ would be 3, 6, 9 and $12 \mathrm{~dB}$. The second method recognises that the interval between 1 and $2 \mathrm{kHz}$ is an octave, whereas the remaining intervals are half octaves. Therefore, using the same example, the interpolated values would be 5, 7.5, 10 and $12.5 \mathrm{~dB}$. The second method would yield a straight line if plotted on a conventional audiogram. We have used the second method, sometimes referred to as 'logarithmic interpolation'; that method should be used when the present procedure is used for quantification of noise-induced hearing loss.

For those wishing to use a calculator or spreadsheet, the formulae to use are as follows, where $m_{1 k}$ is the misfit at $1 \mathrm{kHz}, m_{8 k}$ is the misfit at $8 \mathrm{kHz}$, and $m_{2 k}, m_{3 k}, m_{4 k}$ and $m_{6 k}$ are the interpolated misfit values.

$$
\begin{gathered}
m_{2 k}=\left(m_{1 k} \times 0.67\right)+\left(m_{8 k} \times 0.33\right) \\
m_{3 k}=\left(m_{1 k} \times 0.5\right)+\left(m_{8 k} \times 0.5\right) \\
m_{4 k}=\left(m_{1 k} \times 0.33\right)+\left(m_{8 k} \times 0.67\right) \\
m_{6 k}=\left(m_{1 k} \times 0.17\right)+\left(m_{8 k} \times 0.83\right)
\end{gathered}
$$

\section{Selection of age-associated hearing loss statistic}

In some cases, there is more than one age-associated hearing loss statistic that could reasonably be selected for the diagnostic phase of the calculations (Pass One) and/or for the quantification phase (Pass Two). These will generally yield slightly different results, although seldom sufficiently different to lead to a categorically different outcome. This flexibility offers the temptation to fine-tune the analysis in order to reach a particular conclusion, which is undesirable and places too much emphasis on small numerical differences rather than looking at the whole clinical picture. Therefore, we recommend that the range of selected ageassociated hearing loss statistics should be restricted. The selected age-associated hearing loss statistic should be for the claimant's age at the time of examination, or the nearest age on the chosen database, which may be in intervals of 5 years, as in the original Guidelines. By default, the median should be selected, unless an alternative percentile clearly gives a better fit at the anchor points (based on the sum of the differences between the anchor point values and the ageassociated hearing loss values at the same frequencies). Tables 2 and 3 of the original Guidelines give the 25th and 75 th percentiles as well as the median (50th percentile). Other percentiles can be calculated by reference to ISO 7029 and application of the correction factors given in the legends of the tables in the Guidelines.

Note that these recommendations apply separately to each ear and to Pass One and Pass Two age-associated hearing loss selections. Unexplained sensorineural hearing loss asymmetries occur commonly in the general population ${ }^{13}$, and so $\mathbf{6}$ different age-associated hearing loss statistics may be appropriate for the left and right ears.

\section{Selection of upper anchor-point frequency}

The usual upper anchor-point frequency is $8 \mathrm{kHz}$. In some cases, the threshold at $8 \mathrm{kHz}$ is clearly out of line with the general trend of age-associated hearing loss and this situation is referred to in the Guidelines as a 'precipitous fall-off. In such circumstances, the Guidelines allow selection of $6 \mathrm{kHz}$ as an alternative upper anchor-point frequency. However, the method of quantification described here requires use of $8 \mathrm{kHz}$ as the upper anchor point and therefore, use of $6 \mathrm{kHz}$ as the upper anchor point is no longer recommended.

Nonetheless, cases will arise where the threshold at $8 \mathrm{kHz}$ is clearly out of line with the trend for age-associated hearing loss and an alternative approach is required. In such circumstances, it is recommended that the user of the Guidelines should select a threshold value at $8 \mathrm{kHz}$ that is in line with the overall trend for age-associated hearing loss, instead of the measured value, to use in the calculations. That might be achieved by plotting the audiogram against percentile curves for age-associated hearing loss and extrapolating from the thresholds at lower frequencies. While it is recognised that this is somewhat arbitrary and inimical to a formulaic approach, it should only be required in a small minority of cases. This underlines the fact that the Guidelines are merely a guide and need to be supplemented by careful and objective clinical judgement. 